\title{
Acute Kidney Disease Increases the Risk of Post-Kidney Biopsy Bleeding Complications
}

\author{
Jing Xu Xiaojing Wu Yaowen Xu Hong Ren Weiming Wang \\ Weihong Chen Pingyan Shen Xiao Li Hao Shi Jingyuan Xie \\ Xiaonong Chen Wen Zhang Xiaoxia Pan \\ Department of Nephrology, Ruijin Hospital, Shanghai JiaoTong University School of \\ Medicine, Shanghai, China
}

\section{Keywords}

Kidney biopsy $\cdot$ Acute kidney disease $\cdot$ Bleeding $\cdot$ Complications

\begin{abstract}
Introduction: Kidney biopsy, providing the insightful information for most kidney diseases, is an invasive diagnostic tool with certain risks ranging from the least severe macroscopic hematuria to the most severe life-threatening bleeding necessitating renal artery embolization. We aimed to compare the postbiopsy bleeding complications between 2 common methods and to further explore the risk factors of bleeding complications in patients using the negative pressure suction puncture (NPS) method. Methods: We retrospectively collected the data from percutaneous native kidney biopsies in 2016. The clinical, laboratory tests, pathological findings, and the occurrence of bleeding complications following kidney biopsy were analyzed. The kidney biopsy was performed in our center by experienced nephrologists with 2 different methods, namely, NPS method and real-time ultrasound-guided needle (RTU) method. We compared rates of complications between 2 methods and evaluated univariate and multivariate association of risk factors with bleeding complications in the NPS group. Results: 626 kidney biopsies were performed between January 2016 and December 2016. There were $83.2 \%(521 / 626)$ participants in the NPS group and $16.8 \%(105 / 626)$ in the RTU group. There were more participants in the RTU group needing $>1$ needle pass during biopsy than those in the NPS group ( 61.0 vs. $14.7 \%, p<0.001)$. Acute kidney disease (AKD) occurred before the procedure of kidney biopsy accounted for $13.8 \%$ (72/521) in the NPS group and 1.9\% (2/105) in the RTU group. The renal pathological findings revealed higher number of glomeruli in the NPS group than in the RTU group ( $26.8 \pm 13.0$ vs. $17.2 \pm 8.6, p<0.001)$. The incidence of bleed-
\end{abstract}


ing complications in the NPS group was lower than that in the RTU group $(9.2$ vs. $21.9 \%, p<$ 0.01). Logistic multivariate regression showed that AKD was independently associated with bleeding complications after kidney biopsy in the NPS group. Conclusion: Regarding the bleeding risk, there was noninferiority of NPS over RTU. AKD contributes to higher risks of bleeding complications after kidney biopsy.

\section{Introduction}

Kidney biopsy is an essential method for the diagnosis as well as the assessment of treatment and prognosis for various kidney diseases. As the gold standard of diagnosis in glomerular diseases, tubular diseases, renal vascular diseases, and other secondary kidney diseases, kidney biopsy plays an irreplaceable role. According to the personal experience of nephrologists or radiologists and specific technical support conditions, different methods of percutaneous kidney biopsy have been widely used in different nephrology units [1]. Despite the overall safety of this invasive procedure, not many but few patients may still suffer from postbiopsy bleeding complications. These complications vary from the least severe macroscopic hematuria to the most severe ones requiring blood transfusion or embolization therapy. Several previous studies had shown that 1-deamino-8-D-arginine vasopressin (DDAVP) infusion might be effective to prevent postbiopsy bleeding complications. However, Lim et al. [2] conducted a systemic review of the related clinical trials and claimed that there was insufficient high-quality evidence to support the routine use of DDAVP prior to percutaneous kidney biopsies. Regarding the role of needle size, a retrospective study [3] demonstrated 16-G needles did not have a significant increase in bleeding complications compared with 18-G needles. Although several studies have revealed other risk factors associated with bleeding [4-6], there is no optimal way to accurately identify patients who will develop postbiopsy complications. To maximize the number of glomeruli in kidney biopsy samples and reduce the risk of major bleeding complications have always been the concern of nephrologists in current clinical practice. Three techniques are currently used for radiological guidance: real-time ultrasonographic (US) guidance, US assessment before kidney biopsy, and computed tomography (CT) guidance. In recent years, most centers use real-time ultrasound-guided needle (RTU) biopsy due to its fast learning curve, while negative pressure suction puncture (NPS) method is much less used. Our center still incorporates the NPS biopsy and RTU biopsy for fellow training. The current study was conducted to compare the postbiopsy bleeding complications between these 2 methods and to further explore the risk factors of bleeding complications in patients using the negative pressure suction puncture method.

\section{Methods}

\section{Participants}

This retrospective study was performed using the data of patients who underwent kidney biopsy in Ruijin Hospital affiliated to Shanghai Jiao Tong University School of Medicine from January 2016 to December 2016. All participants received oral and written information about the study and signed an informed consent. This study was approved by the Ethics Committee of Ruijin Hospital affiliated to Shanghai Jiao Tong University School of Medicine. Age at biopsy, gender, clinical and laboratory data, number of needle passes, number of glomeruli, histo- 


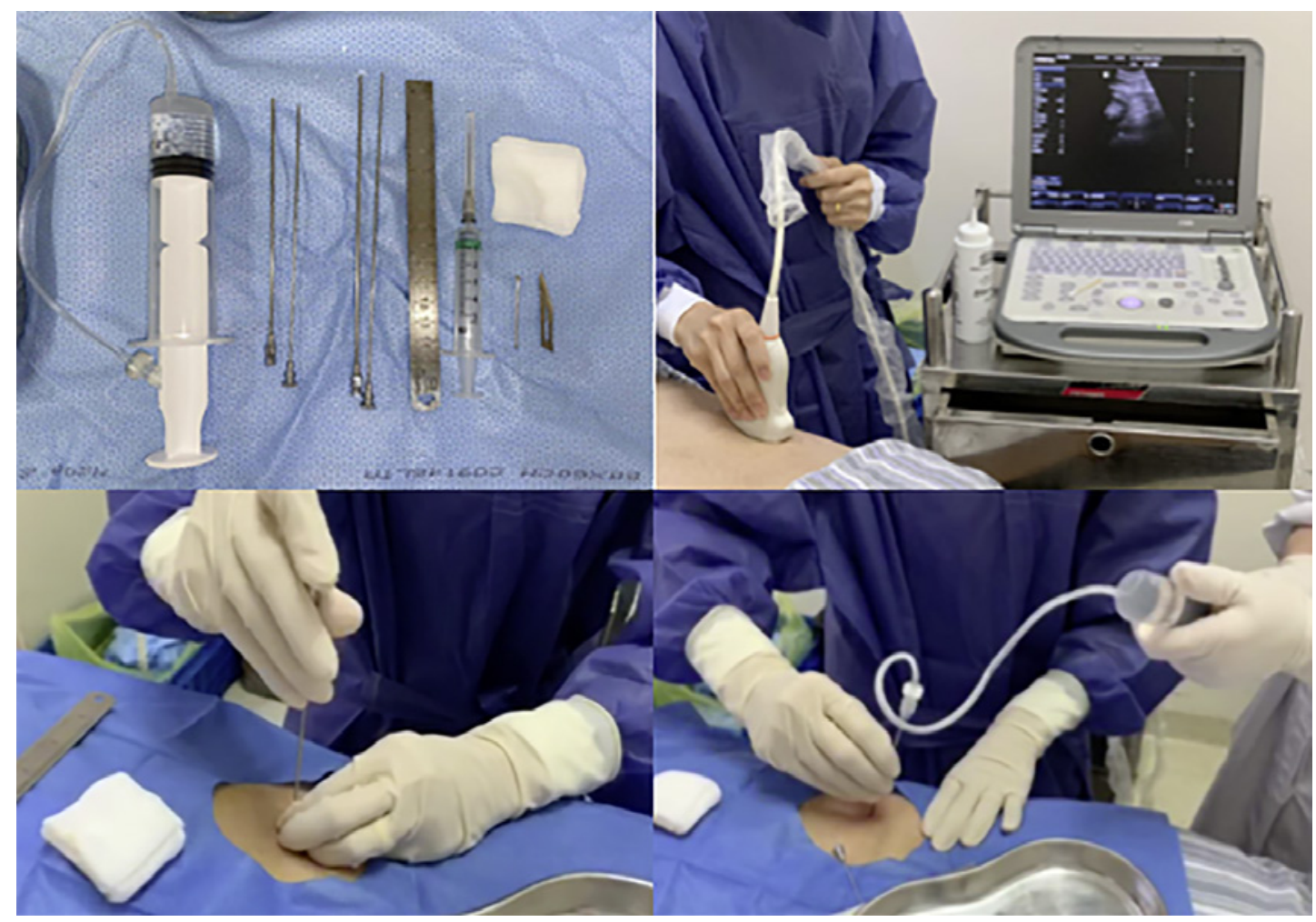

Fig. 1. Images of the instrument and procedure of the NPS method. NPS, negative pressure suction puncture.

pathological diagnosis, and the occurrence of postbiopsy bleeding adverse events (macroscopic hematuria, perirenal hematoma, and renal artery embolization) were collected.

\section{Kidney Biopsy Procedures}

All kidney biopsies were performed by 2 experienced nephrologists and 2 radiologists. The patients were kept in the prone position. A hard sandbag was padded under the abdomen to reduce kidney movement during kidney puncture. A routine preliminary USG with a 3.5-MHz transducer had scanned both the kidneys for complete evaluation of cortical and sinus echogenicity by longitudinal and transverse images and exclusion of any structural abnormalities prior to the biopsy. The bottom of the kidney was designated to be the puncture point. (1) NPS method: for blind biopsy, USG surface marking for kidney biopsy over the lower pole of the right kidney by default was carried out and the perpendicular distance from the skin to the kidney cortex was measured. The skin was disinfected and draped and anesthetized with $1 \%$ lignocaine. After the needle reached the kidney capsule, the core of the needle was pulled out, the needle bolt was placed in the needle tube, and a syringe $(50 \mathrm{~mL})$ was connected. The needle was quickly inserted into the kidney, and the assistant produced negative pressure to draw the kidney tissue (Fig. 1). (2) RTU method: the USG probe was covered with a sterile cover. Local anesthesia with $1 \%$ lignocaine was injected under USG guidance from the skin to the kidney capsule. The Bard gun was advanced under USG guidance along the anesthetized tract. Biopsies were performed with a biopsy adapter. After the kidney capsule was reached, the patient held his breath and the trigger was pulled. The number of needle passes was 1 or 2. Sixteen-gauge needles were used in our study. Patients were kept in bed rest for $24 \mathrm{~h}$ and observed for postprocedure hematuria, and US or CT scan was performed between 1 and 3 days to diagnose perirenal hematoma. 
Table 1. Characteristics of participants using 2 methods of kidney biopsy

\begin{tabular}{|c|c|c|c|}
\hline & $\begin{array}{l}\text { NPS group } \\
(n=521 ; 83.2 \%)\end{array}$ & $\begin{array}{l}\text { RTU group } \\
(n=105 ; 16.8 \%)\end{array}$ & $p$ value \\
\hline \multicolumn{4}{|l|}{ Clinical characteristics } \\
\hline Men, $n(\%)$ & $277(53.2)$ & $57(54.3)$ & 0.83 \\
\hline Age, years & 43 (14-81) & $38(17-73)$ & 0.17 \\
\hline Nephrotic syndrome, $n(\%)$ & $223(42.8)$ & $36(34.3)$ & 0.1 \\
\hline Diabetes, $n(\%)$ & $46(8.8)$ & $8(7.6)$ & 0.7 \\
\hline Hypertension, $n(\%)$ & $211(40.5)$ & $49(46.7)$ & 0.24 \\
\hline SBP, mm Hg & $128.6 \pm 17.2$ & $133.1 \pm 15.1$ & 0.04 \\
\hline $\mathrm{DBP}, \mathrm{mm} \mathrm{Hg}$ & $77.3 \pm 10.8$ & $82.1 \pm 9.6$ & 0.27 \\
\hline Height, $\mathrm{cm}$ & $166.1 \pm 8.2$ & $165.5 \pm 7.7$ & 0.56 \\
\hline Weight, kg & $66.0 \pm 13.9$ & $65.0 \pm 12.0$ & 0.22 \\
\hline BMI, $\mathrm{kg} / \mathrm{m}^{2}$ & $23.8 \pm 4.0$ & $23.6 \pm 3.3$ & 0.70 \\
\hline Dialysis, $n(\%)$ & $20(3.8)$ & $0(0)$ & 0.03 \\
\hline Macroscopic hematuria, $n(\%)$ & $34(6.5)$ & $3(2.9)$ & 0.15 \\
\hline AKD, $n(\%)$ & $72(13.8)$ & $2(1.9)$ & $<0.01$ \\
\hline $\mathrm{CKD}, n(\%)$ & $449(86.2)$ & $103(98.1)$ & 0.13 \\
\hline CKD stage $1, n(\%)$ & $209(40.1)$ & $45(42.9)$ & \\
\hline CKD stage $2, n(\%)$ & $120(23.0)$ & $24(22.9)$ & \\
\hline CKD stage $3, n(\%)$ & $82(15.7)$ & $29(27.6)$ & \\
\hline CKD stage $4, n(\%)$ & $28(5.4)$ & $5(4.8)$ & \\
\hline CKD stage $5, n(\%)$ & $10(1.9)$ & $0(0)$ & \\
\hline \multicolumn{4}{|l|}{ Laboratory findings } \\
\hline Serum Cr, $\mu \mathrm{mol} / \mathrm{L}$ & $92.0(40-1,145)$ & $94.0(48-335)$ & 0.66 \\
\hline $\mathrm{eGFR}, \mathrm{mL} / \mathrm{min} / 1.73 \mathrm{~m}^{2}$ & $77.5(3.9-165.9)$ & $79.4(20.1-144.6)$ & 0.39 \\
\hline Proteinuria, g/day & $2.6(0.1-27.3)$ & $2.8(0.04-17.6)$ & 0.85 \\
\hline Albumin, $\mathrm{g} / \mathrm{L}$ & $29.0 \pm 9.5$ & $33.3 \pm 9.3$ & 0.59 \\
\hline Hemoglobin, g/L & $123.0 \pm 22.8$ & $127.4 \pm 21.6$ & 0.83 \\
\hline Monoclonal gammopathy, $n(\%)$ & $25(4.8)$ & $3(2.9)$ & 0.60 \\
\hline \multicolumn{4}{|l|}{ Kidney size, $n(\%)$} \\
\hline Normal, $>100 \mathrm{~mm}$ & $439(84.3)$ & $93(88.6)$ & 0.5 \\
\hline Small, $90-100 \mathrm{~mm}$ & $80(15.4)$ & $12(11.4)$ & \\
\hline Malformation & $2(0.4)$ & $0(0)$ & \\
\hline Needle passes $>1, n(\%)$ & $92(14.7)$ & $64(61.0)$ & $<0.001$ \\
\hline \multicolumn{4}{|l|}{ Biopsy findings } \\
\hline Glomeruli, $n(\%)$ & $26.8(13.0)$ & $16.0(7.9)$ & $<0.001$ \\
\hline Samples without glomeruli, $n(\%)$ & $1(0.2)$ & 0 & 1.0 \\
\hline Proportion of global glomerulosclerosis & $10(0-100)$ & $13(0-85)$ & 0.87 \\
\hline \multicolumn{4}{|l|}{ Increase in the proportion of global glomerulosclerosis, } \\
\hline$n(\%)$ (age adjustment) & $262(50.3)$ & $58(55.2)$ & 0.36 \\
\hline \multicolumn{4}{|l|}{ Interstitial fibrosis, $n(\%)$} \\
\hline None $(0)$ & $50(9.6)$ & $14(13.3)$ & 0.088 \\
\hline Mild $(<25)$ & $337(64.9)$ & $58(55.2)$ & \\
\hline Moderate (26-50) & $76(14.6)$ & $24(22.9)$ & \\
\hline Severe $(>50)$ & $56(10.8)$ & $9(8.6)$ & \\
\hline \multicolumn{4}{|l|}{ Interstitial inflammation, $n(\%)$} \\
\hline None $(0)$ & $54(10.4)$ & $15(14.3)$ & 0.12 \\
\hline Mild $(<25)$ & $298(57.2)$ & $54(51.4)$ & \\
\hline Moderate (26-50) & $82(15.7)$ & $24(22.9)$ & \\
\hline Severe $(>50)$ & $87(16.7)$ & $12(11.4)$ & \\
\hline \multicolumn{4}{|l|}{ Tubular atrophy, $n(\%)$} \\
\hline None $(0)$ & $41(7.9)$ & $10(9.5)$ & 0.32 \\
\hline Mild (<25) & $340(65.3)$ & $62(59.0)$ & \\
\hline Moderate (26-50) & $84(16.1)$ & $24(22.9)$ & \\
\hline Severe $(>50)$ & $56(10.7)$ & $9(8.6)$ & \\
\hline Arteriosclerosis & $277(53.2)$ & $53(50.5)$ & 0.67 \\
\hline
\end{tabular}

Values are expressed as mean $\pm \mathrm{SD}$, median (range), $n(\%)$, or as otherwise indicated. NPS, negative pressure suction puncture; RTU, real-time ultrasound guided needle; SBP, systolic blood pressure; DBP, diastolic blood pressure; BMI, body mass index; eGFR, estimated glomerular filtration rate. 


\section{Definitions}

Hypertension was defined as blood pressure $\geq 140 / 90 \mathrm{~mm} \mathrm{Hg}$.

Perirenal hematoma: we defined the presence of perirenal hematoma as a hematoma on imaging that was over $1 \mathrm{~cm}$ in any dimension [7].

Number of glomeruli: it referred to the sum of light microscopy, immunofluorescence, and electron microscope.

Acute kidney disease (AKD): we used the definition of AKD according to the Kidney Disease: Improving Global Outcomes (KDIGO) guidelines. AKD includes acute kidney injury (AKI) or subacute decreases in glomerular filtration rate (GFR) (i.e., GFR $<60 \mathrm{~mL} / \mathrm{min} / 1.73$ $\mathrm{m}^{2}$ for $<3$ months or decrease in GFR by $\geq 35 \%$ or increase in serum $\mathrm{Cr}$ by $>50 \%$ for $<3$ months) $[8,9]$.

\section{Statistical Analyses}

SPSS software, version 21.0 (IBM, Chicago, IL, USA), was used for statistical analyses. Quantitative data were expressed as mean \pm SD or median with range. Categorical data were expressed as frequency (percentage). For comparison of clinical and pathological parameters among different groups, nonparametric variables were analyzed by the Mann-Whitney test or the Kruskal-Wallis test and categorical variables were analyzed by Pearson's two-tailed test or Fisher's exact test, whenever appropriate. To determine the risk factors of postbiopsy bleeding complications, we used univariate logistic regression analysis followed by multivariate logistic regression with stepwise backward inclusion of variables to minimize the number of covariates in the model. $p$ values were 2 sided, and $p<0.05$ was considered to indicate statistical significance.

\section{Results}

\section{Baseline Characteristics}

Basic characteristics of participants of this study undergoing 2 different methods of kidney biopsy (NPS and RTU) are shown in Table 1. There was no significant difference prior to kidney biopsy between 2 groups in gender, age, percentage of nephrotic syndrome, history of diabetes or hypertension, diastolic blood pressure, height, weight, BMI, Cr, estimated glomerular filtration rate (eGFR), proteinuria, serum albumin, hemoglobin, monoclonal protein, kidney size, dialysis treatment, and incidence of macroscopic hematuria. The systolic blood pressure prior to biopsy in the NPS group was slightly lower than that in the RTU group (128.6 \pm 17.2 vs. $133.1 \pm 15.1 \mathrm{~mm} \mathrm{Hg}, p=0.04$ ). There were more participants in the RTU group needing $>1$ needle pass during biopsy than those in the NPS group ( 61.0 vs. $14.7 \%, p<0.001$ ).

The classification of AKD (including AKI) and chronic kidney disease (CKD) was based on the KDIGO guidelines on the classification of AKI $[8,9]$ and CKD [10]. AKD patients accounted for $13.8 \%(72 / 521)$ in the NPS group and $1.9 \%(2 / 105)$ in the RTU group $(p<0.01)$. There was no significant difference of prevalence of CKD between 2 groups.

The renal pathological findings showed that the average number of glomeruli was higher in the NPS group than that in the RTU group $(26.8 \pm 13.0$ vs. $17.2 \pm 8.6, p<0.001)$. There was only 1 sample without glomeruli in the NPS group. There was no significant difference between the 2 groups regarding the proportion of global glomerulosclerosis, the degree of tubulointerstitial lesions, and arteriosclerosis. Detailed pathological diagnosis of the 2 groups is shown in online suppl.Fig.1;forallonlinesuppl.material,seewww.karger.com/doi/10.1159/000509443. The distribution of pathological diagnosis of secondary kidney diseases in AKD patients in the NPS group is shown in Figure 2. The scatterplot of the number of glomeruli sampled in patients with and without bleeding complications is shown in Figure 3. 
Kidney and

Blood Pressure

Research

\begin{tabular}{l|l}
\hline Kidney Blood Press Res 2020;45:873-882 \\
\hline DOI: 10.1159/000509443 & $\begin{array}{l}\text { @ } 2020 \text { The Author(s). Published by S. Karger AG, Basel } \\
\text { www.karger.com/kbr }\end{array}$ \\
\hline
\end{tabular}

Xu et al.: Acute Kidney Disease Correlates with Higher Postbiopsy Bleeding

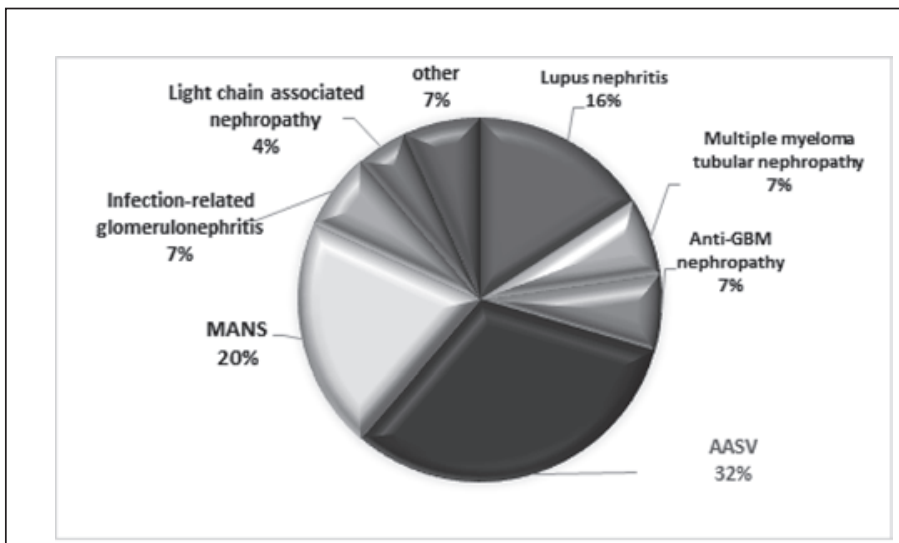

GBM: glomerular basement membrane, AASV: anti-neutrophil cytoplasmic antibody-associated systemic vasculitis, MANS: malignant hypertension

phil cytoplasmic antibody-associated systemic vasculitis; MANS, malignant hypertension.

Fig. 2. Pathological distribution of secondary kidney diseases in AKD patients in the NPS group. AKD, acute kidney disease; NPS, negative pressure suction puncture; GBM, glomerular basement membrane; AASV, anti-neutro-

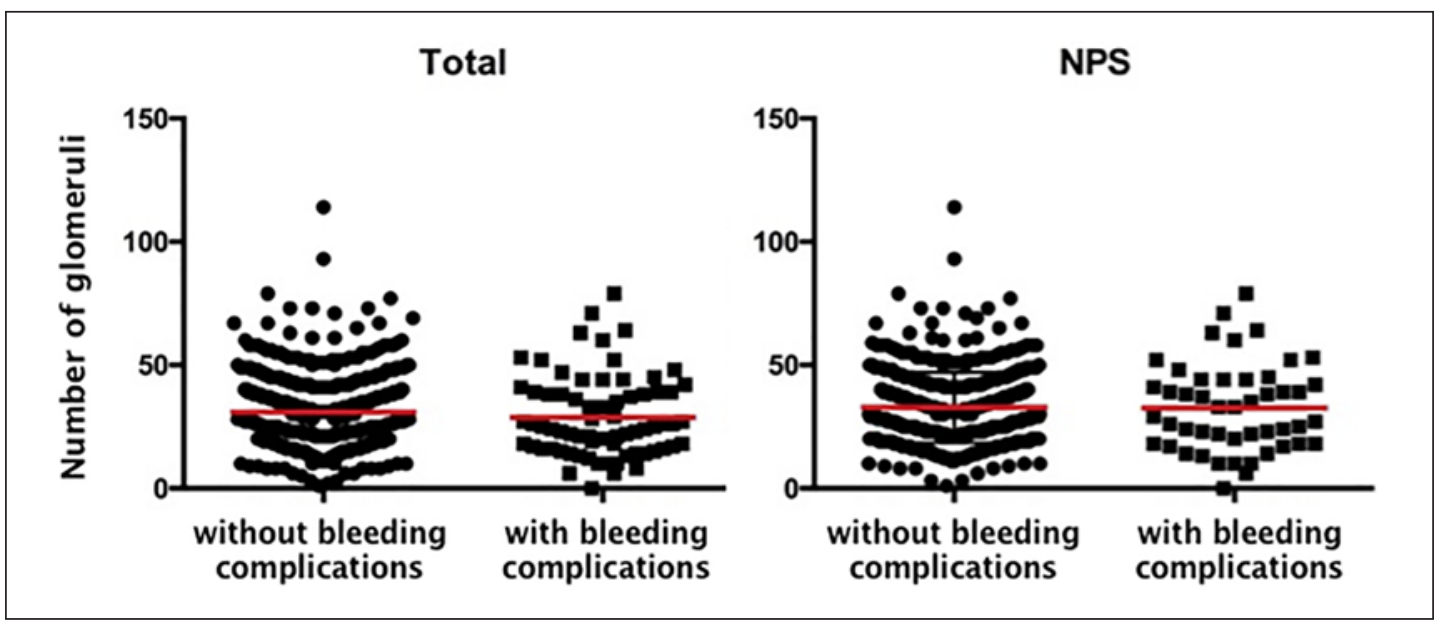

Fig. 3. Scatterplot of the number of glomeruli sampled in patients with and without bleeding complications. NPS, negative pressure suction puncture.

\section{Postbiopsy Bleeding Complications}

The incidence of bleeding complications in the NPS group was $9.2 \%(48 / 521)$, including $50 \%$ (24/48) of macroscopic hematuria alone, 39.6\% (19/48) of perirenal hematoma alone, and $10.4 \%$ (5/48) of macroscopic hematuria and hematoma. $0.77 \%(4 / 521)$ of patients with post-kidney biopsy bleeding complications in the NPS group needed the renal artery embolization. The incidence of bleeding complications in the RTU group was $21.9 \%$ (23/105), among which $26.1 \%$ (6/23) were macroscopic hematuria alone, $73.9 \%(17 / 23)$ were hematoma alone, and none needed renal artery embolization. The patients with postbiopsy bleeding complications in the NPS group had higher proportion of AKD than those without bleeding complications ( 25.0 vs. $12.7 \%, p=0.02)$. The lower hemoglobin $(\mathrm{g} / \mathrm{L})$ was found in the patients with bleeding complications than those without bleeding complications $(115.7 \pm$ 23.6 vs. $123.7 \pm 22.6, p=0.02$ ). There was also less overweight or obesity in the patients with bleeding complications than those without bleeding complications ( 29.2 vs. $44.8 \%, p=0.04$ ) (Table 2). 
Table 2. Comparing the patients with or without post biopsy bleeding complications in the NPS group

\begin{tabular}{|c|c|c|c|}
\hline & $\begin{array}{l}\text { With bleeding } \\
(n=48 ; 9.2 \%)\end{array}$ & $\begin{array}{l}\text { Without bleeding } \\
(n=473 ; 90.8 \%)\end{array}$ & $p$ value \\
\hline \multicolumn{4}{|l|}{ Clinical characteristics } \\
\hline Men, $n(\%)$ & $20(41.7)$ & $257(54.3)$ & 0.09 \\
\hline Age, years & 47 (18-79) & $42(14-81)$ & 0.19 \\
\hline Nephrotic syndrome, $n(\%)$ & $16(33.3)$ & $207(43.8)$ & 0.17 \\
\hline Diabetes, $n(\%)$ & $5(10.4)$ & $41(8.7)$ & 0.68 \\
\hline Hypertension, $n(\%)$ & $22(45.8)$ & $189(40.0)$ & 0.43 \\
\hline Uncontrolled hypertension, $n(\%)$ & $12(25.0)$ & $107(22.6)$ & 0.71 \\
\hline Dialysis, $n(\%)$ & $3(6.2)$ & $18(3.8)$ & 0.43 \\
\hline Needle passes $>1, n(\%)$ & $12(25.0)$ & $80(16.9)$ & 0.16 \\
\hline Acute kidney disease, $n(\%)$ & $12(25.0)$ & $60(12.7)$ & 0.02 \\
\hline Overweight or obesity, $n(\%)$ & $14(29.2)$ & $212(44.8)$ & 0.04 \\
\hline \multicolumn{4}{|l|}{ Laboratory findings } \\
\hline Hemoglobin, g/L & $115.7 \pm 23.6$ & $123.7 \pm 22.6$ & 0.02 \\
\hline EPI-eGFR $<60 \mathrm{~mL} / \mathrm{min} / 1.73 \mathrm{~m}^{2}, n(\%)$ & $19(39.6)$ & $171(36.2)$ & 0.64 \\
\hline \multicolumn{4}{|l|}{ Biopsy findings } \\
\hline Glomeruli & $31(0-79)$ & $31(1-114)$ & 0.69 \\
\hline Increase in the proportion of glomerulosclerosis, $n(\%)$ & $21(43.8)$ & $241(51.0)$ & 0.34 \\
\hline Tubulointerstitial changes $>25 \%, n(\%)$ & $13(27.1)$ & $120(25.4)$ & 0.86 \\
\hline Interstitial inflammation $>25 \%, n(\%)$ & $16(33.3)$ & $153(32.3)$ & 0.87 \\
\hline Renal arteriolosclerosis, $n(\%)$ & $27(56.2)$ & $250(52.9)$ & 0.65 \\
\hline
\end{tabular}

Table 3. Logistic regression analysis of high-risk factors for postbiopsy bleeding complications in the NPS group

\begin{tabular}{|c|c|c|c|c|c|c|}
\hline & \multicolumn{3}{|c|}{ Univariate } & \multicolumn{3}{|c|}{ Multivariate } \\
\hline & OR & $95 \% \mathrm{CI}$ & $p$ value & OR & $95 \% \mathrm{CI}$ & $p$ value \\
\hline Age, years & 1.012 & $0.994-1.031$ & 0.19 & & & \\
\hline Sex (male, ref.) & 1.666 & $0.913-3.040$ & 0.10 & & & \\
\hline Nephrotic syndrome & 0.643 & $0.343-1.203$ & 0.17 & & & \\
\hline Diabetes & 1.225 & $0.460-3.264$ & 0.69 & & & \\
\hline Hypertension & 1.271 & $0.700-2.309$ & 0.43 & & & \\
\hline Overweight or obesity & 0.507 & $0.265-0.969$ & 0.04 & 0.525 & $0.273-1.006$ & 0.052 \\
\hline Dialysis & 1.685 & $0.478-5.941$ & 0.42 & & & \\
\hline Needle passes, $n$ & 0.611 & $0.304-1.225$ & 0.17 & & & \\
\hline Serum $\mathrm{Cr}, \mu \mathrm{mol} / \mathrm{L}$ & 1.001 & $0.999-1.003$ & 0.18 & & & \\
\hline AKD & 2.294 & $1.131-4.654$ & 0.02 & 2.196 & $1.078-4.475$ & 0.03 \\
\hline Hemoglobin, g/L & 0.985 & $0.973-0.998$ & 0.02 & & & \\
\hline Kidney size (normal size ref.) & 1.683 & $0.820-3.454$ & 0.16 & & & \\
\hline Glomeruli, $n$ & 0.999 & $0.979-1.020$ & 0.91 & & & \\
\hline Increase in the proportion of glomerulosclerosis, $\%$ & 0.749 & $0.412-1.362$ & 0.34 & & & \\
\hline Tubulointerstitial changes, $\%$ & 1.093 & $0.559-2.134$ & 0.80 & & & \\
\hline Interstitial inflammation, $\%$ & 1.046 & $0.557-1.964$ & 0.89 & & & \\
\hline Renal arteriolosclerosis, \% & 1.147 & $0.631-2.086$ & 0.65 & & & \\
\hline
\end{tabular}

NPS, negative pressure suction puncture; AKD, acute kidney disease. 


\section{AKD Contributes to More Postbiopsy Bleeding Complications}

There were only 2 patients with AKD in the RTU group, so we only conducted the logistic univariate regression analysis in the NPS group to analyze the potential risk factors of postbiopsy bleeding complications. In the univariate analysis, we found that nonoverweight or obesity, hemoglobin, and AKD were significantly associated with bleeding complications. The multivariate regression analysis showed that only AKD was associated with postbiopsy bleeding complications (Table 3).

\section{Discussion}

In most previous studies, the RTU kidney biopsy was the preferred technique [11]. It was reported that there was a lower mean number of glomeruli in biopsies performed with realtime ultrasound guidance than that with blind biopsies in 1998 [12]. In 2014, another Korea study showed no difference of the tissue yield between blind $(n=271)$ and US-guided $(n=$ 170) kidney biopsy performed by nephrologists [13]. In a French retrospective study of 943 kidney biopsies comparing 5 different practices, they found no difference concerning severe complications for different biopsy procedures, but the mean number of glomeruli in the $\mathrm{C} 1$ group (US assessment before kidney biopsy) was higher than that in the C4 group (real-time US guidance by nephrologists) [14]. In our study, the NPS group had higher average number of glomeruli $(26.8 \pm 13.0$ vs. $17.2 \pm 8.6, p<0.001)$ and less needle pass $(14.7$ vs. $61.0 \%, p<$ 0.001 ) than the RTU group. Although this difference could be explained by the experience and discretion of nephrologists, it shows that the NPS method for kidney biopsy is not inferior to the RTU biopsy.

The most common postbiopsy complications are macroscopic hematuria, perirenal hematomas, arteriovenous fistulas, and injuries to blood vessels and surrounding organs [15, 16]. The reported occurrence of complications following a kidney biopsy tends to vary from one region to another. A study from China reported the incidence of severe bleeding to be $0.39 \%$ out of 3,577 native kidney biopsies [17]. However, much higher rates have been reported in other parts of the world. The incidence of bleeding after kidney biopsy ranges from 13 to $34 \%[18,19]$. Percutaneous kidney biopsy is associated with bleeding complications such as macroscopic hematuria (3.5\%), postbiopsy hematoma (11.6\%), erythrocyte transfusion $(0.9 \%)$, and rarely nephrectomy $(0.01 \%)$ or death $(0.02 \%)$ in a meta-analysis [20]. In our study, the incidence of total bleeding complications was $9.2 \%(48 / 521)$ in the NPS group and $21.9 \%(23 / 105)$ in the RTU group. Though the NPS group had lower rate of bleeding complications than the RTU group, $0.77 \%$ (4/521) patients in the NPS group developed lifethreatening active bleeding requiring the renal artery embolization, while the RTU group had none.

Prior studies have shown that risk factors of bleeding complications were hypertension, thrombocytopenia, and coagulopathy prior to the procedure. Unmodifiable risk factors such as poor kidney function, gender, and underlying histological diagnosis may be used to identify high-risk patients [6]. Other studies found that hospitalized patients receiving the kidney biopsy for AKD have a higher risk of requiring the blood transfusion after the procedure [20, 21]. Our study showed that AKD was associated with higher postbiopsy bleeding after kidney biopsy using the NPS method. Despite the unknown mechanism, we suggest that patients with AKD prior to biopsy be more tightly monitored after kidney biopsy.

The strength of this study is the in-depth analysis of risk factors for postbiopsy bleeding complications in patients receiving kidney biopsy using the NPS method. This study was carried out in inpatient setting, and patients benefit from a prolonged surveillance $(24 \mathrm{~h})$ in the hospital after kidney biopsy. Our study has some limitations. This is a single-center 
study with substantial experience with the NPS method for kidney biopsy and >600 kidney biopsies per year in a tertiary teaching hospital. The results may not be generalizable for other centers.

\section{Conclusions}

The NPS method for kidney biopsy is not inferior to the RTU biopsy. We could get more number of glomeruli with less needle pass. This may be associated with more training of nephrologists and warrants further study. AKD is an independent risk factor of bleeding complications after kidney biopsy using the NPS method. The underlying mechanism is unknown. A well-designed prospective study would provide with more insights.

\section{Statement of Ethics}

All participants received oral and written information about the study and signed an informed consent. This study was approved by the Ethics Committee of Ruijin Hospital affiliated to Shanghai Jiao Tong University School of Medicine.

\section{Conflict of Interest Statement}

All authors declare that they have no financial or personal conflicts of interest.

\section{Funding Sources}

This study was supported by the National Key Research and Development Program of China (Grant No. 2016YFC1305402), Shanghai Municipal Key Clinical Specialty (shslczdzk 02502), and National Natural Science Foundation of China (30871001).

\section{Author Contributions}

J.X. contributed to the research aims; data acquisition, analysis, and interpretation; writing of the manuscript; and drafting the article. X.P. and W.Z. designed the study and its concept and contributed to interpretation of the data and writing of the manuscript. X.W. contributed to data acquisition, analysis, and interpretation. Y.X., H.R., W.W., P.S., X.L., H.S., J.X., and X.C. contributed to interpretation of the data. W.C. contributed to acquisition of data. All authors contributed to the drafting of the article, revising it critically, and finally approving the version to be submitted.

\section{References}

1 Bollee G, Martinez F, Moulin B, Meulders Q, Rougier JP, Baumelou A, et al. Renal biopsy practice in France: results of a nationwide study. Nephrol Dial Transplant. 2010;25(11):3579-85.

2 Lim CC, Tan HZ, Tan CS, Healy H, Choo J, Gois PHF. Desmopressin acetate (DDAVP) to prevent bleeding in percutaneous kidney biopsy: a systematic review. Intern Med J. 2020.

3 Mai J, Yong J, Dixson H, Makris A, Aravindan A, Suranyi MG, et al. Is bigger better? A retrospective analysis of native renal biopsies with 16 Gauge versus 18 Gauge automatic needles. Nephrology. 2013;18(7):525-30. 
4 Shidham GB, Siddiqi N, Beres JA, Logan B, Nagaraja HN, Shidham SG, et al. Clinical risk factors associated with bleeding after native kidney biopsy. Nephrology. 2005;10(3):305-10.

5 Kriegshauser JS, Patel MD, Young SW, Chen F, Eversman WG, Chang YH. Risk of bleeding after native renal biopsy as a function of preprocedural systolic and diastolic blood pressure. J Vasc Interv Radiol. 2015;26(2): 206-12.

6 Bakdash K, Schramm KM, Annam A, Brown M, Kondo K, Lindquist JD. Complications of percutaneous renal biopsy. Semin Intervent Radiol. 2019;36(2):97-103.

7 Cui S, Heller HT, Waikar SS, McMahon GM. Needle size and the risk of kidney biopsy bleeding complications. Kidney Int Rep. 2016;1(4):324-6.

8 Palevsky PM, Liu KD, Brophy PD, Chawla LS, Parikh CR, Thakar CV, et al. KDOQI US commentary on the 2012 KDIGO clinical practice guideline for acute kidney injury. Am J Kidney Dis. 2013;61(5):649-72.

9 Covic A, Schiller A, Volovat C, Gluhovschi G, Gusbeth-Tatomir P, Petrica L, et al. Epidemiology of renal disease in Romania: a 10 year review of two regional renal biopsy databases. Nephrol Dial Transplant. 2006;21(2): 419-24.

10 Hanko JB, Mullan RN, O’Rourke DM, McNamee PT, Maxwell AP, Courtney AE. The changing pattern of adult primary glomerular disease. Nephrol Dial Transplant. 2009;24(10):3050-4.

11 Maya ID, Maddela P, Barker J, Allon M. Percutaneous renal biopsy: comparison of blind and real-time ultrasound-guided technique. Semin Dial. 2007;20(4):355-8.

12 Kim D, Kim H, Shin G, Ku S, Ma K, Shin S, et al. A randomized, prospective, comparative study of manual and automated renal biopsies. Am J Kidney Dis. 1998;32(3):426-31.

13 Chung S, Koh ES, Kim SJ, Yoon HE, Park CW, Chang YS, et al. Safety and tissue yield for percutaneous native kidney biopsy according to practitioner and ultrasound technique. BMC Nephrol. 2014;15:96.

14 Bataille S, Jourde N, Daniel L, Mondain JR, Faure M, Gobert P, et al. Comparative safety and efficiency of five percutaneous kidney biopsy approaches of native kidneys: a multicenter study. Am J Nephrol. 2012;35(5): 387-93.

15 Redfield RR, McCune KR, Rao A, Sadowski E, Hanson M, Kolterman AJ, et al. Nature, timing, and severity of complications from ultrasound-guided percutaneous renal transplant biopsy. Transpl Int. 2016;29(2):16772.

16 Preuss S, Kuechle C, Wagenpfeil S, Schmaderer C, Renders L, Heemann U, et al. Retrospective analysis of ultrasound-detected bleeding complications after ultrasound-guided transcutaneous kidney biopsies. Ultrasound Med Biol. 2017;43(1):153-62.

$17 \mathrm{Xu}$ DM, Chen M, Zhou FD, Zhao MH. Risk factors for severe bleeding complications in percutaneous renal biopsy. Am J Med Sci. 2017;353(3):230-5.

18 Whittier WL, Korbet SM. Timing of complications in percutaneous renal biopsy. J Am Soc Nephrol. 2004;15(1): 142-7.

19 Manno C, Strippoli GF, Arnesano L, Bonifati C, Campobasso N, Gesualdo L, et al. Predictors of bleeding complications in percutaneous ultrasound-guided renal biopsy. Kidney Int. 2004;66(4):1570-7.

20 Corapi KM, Chen JL, Balk EM, Gordon CE. Bleeding complications of native kidney biopsy: a systematic review and meta-analysis. Am J Kidney Dis. 2012;60(1):62-73.

21 Moledina DG, Luciano RL, Kukova L, Chan L, Saha A, Nadkarni G, et al. Kidney biopsy-related complications in hospitalized patients with acute kidney disease. Clin J Am Soc Nephrol. 2018;13(11):1633-40. 